\title{
Kegel Nation App
}

National Cancer Institute

\section{Source}

National Cancer Institute. Kegel Nation App. NCI Thesaurus. Code C155823.

A smartphone-based application designed to help monitor the frequency, progress and quality of a Kegel exercise regimen in both men and women. It allows patients to measure the duration of the pelvic muscle contraction and relaxation by touching the phone's screen during each exercise phase. The app then archives and charts the measured times with a time and date stamp. Patients can also use the app to track other indicators of bladder function recovery, such as urinary urgency and incontinence episodes, pad use, and voiding events. 